\title{
The Effects of Neuromuscular Electrical Stimulation in the Treatment of Stroke
}

\author{
Chen Xueyan ${ }^{1, a}$, Mei Chunli ${ }^{1^{*}, b}$ \\ 1 Beihua University, Jilin, 132031, China \\ aemail:455931074@qq.com, bemail:meixiaoqing2007@126.com
}

(Mei Chunli*is responding author)

\begin{abstract}
Keywords: Neuromuscular electrical stimulation; Stroke; Treatment
Abstract. Stroke is one of the most important factors in the spectrum of the Chinese population. In the current physical therapy for rehabilitation aftermstroke,neuromuscular electrical stimulation therapy is more important. Stroke is a disease with high disability rate. Rehabilitation of stroke patients with hemiplegia is to reduce the severity of disabilities as far as possible, to maximize daily living. At present for the post-stroke paralysis in rehabilitation therapy, neuromuscular electrical stimulation is a widely used method for the treatment of a large number of. Clinical studies have shown that neuromuscular electrical stimulation therapy can significantly improve stroke patients with limb function, improve patient self-care ability, reduce morbidity.
\end{abstract}

\section{Introduction}

Neuromuscular electrical stimulation (Neuromuscular Electric, Stimulation, NMES) belongs to the category of low frequency electric stimulation, the generalized neuromuscular stimulation that can be called NMES, but according to the 2007 Sheffler and Chae, the real NMES refers to lower motor neurons by using low frequency current to stimulate structural integrity, activate or cause muscle contraction improve the current use of transcutaneous electrical nerve stimulation of [1]. as a treatment for muscle function or treatment of neuromuscular disorders (transcutaneous electrical nerve stimulation, TENS) and functional electrical stimulation (functional electrical, stimulation, FES).

\section{Physiological Basis}

Application of low-frequency electrical stimulation of paralyzed limbs in patients with stroke, nerve lesions visible marginal zone cell synapse number, space narrowing, increase the length of synaptic activity, synaptic thickening. Neurite outgrowth after electrical stimulation showed growth trend, the extension direction is roughly consistent with the direction of stimulating electrodes, some cells with the same direction the distant cell axons or dendrites connected and cell processes near a trend of vertical growth, parallel extension. After electrical stimulation of the lesion edge neurons and astrocytes and activity change significantly, the expression of glial fibrillary acidic protein and neurofilament protein expression, expression of microtubule associated protein and neuron apoptosis increased. The lesion vascular density increased, an increase in local blood flow.

Excitatory amino acid toxicity was inhibited, while increasing the expression of brain derived neurotrophic factor, so as to restore neurons, protecting of the brain function [2].

\section{Clinical Application}

Transcutaneous electrical nerve stimulation

TENS will stimulate electrodes placed on the surface of the skin of a patient, the low frequency pulsed DC electrical stimulation of nerve fibers so as to achieve the purpose of.TENS is the treatment of low frequency, high intensity, can cause visible vein muscle contraction.

Width is relatively large $(1 \sim 200 \mathrm{~s})$, the treatment of stimulating electrodes are usually placed on the moving point [3]. 


\section{Treatment of motor dysfunction after stroke}

$\mathrm{Gu}$ Min [4] patients with stroke incidence of TENS within $14 \mathrm{~d}$ treatment, the stimulation frequency was $100 \mathrm{~Hz}, 30 \mathrm{~min}$ for each stimulus, 2 times a day, the stimulus intensity for the patients can tolerate the pain level, a total of 4 weeks, the control group were given electrical stimulation simulation at the same time, the 2 groups of patients received movement learning and training, after the corresponding treatment, the 2 groups of patients with Fugl-Meyer And Barthel index score were statistically significant, that is helpful to improve the motor function of stroke patients underwent TENS treatment as soon as possible, improve the quality of life. Wang Dongyan [5] applications such as low frequency transcutaneous electrical stimulation treatment of 20 cases of patients with wrist function because of the stimulus waveform for the continuous wave, frequency of $50 \mathrm{~Hz}$, with flexion and extension alternate mode, role in muscle, 30min each time, two times a day, 20 days for a course of treatment, the control group routine at the same time, two groups of patients were rehabilitation treatment, the control group and the treatment group before treatment, after the same action MAX, AEMG value of paired $t$ test, $P<0.01$, there were significant differences between the two groups before treatment; after MAX, the AEMG value difference between $t$ test results were significantly different.

\section{Treatment of paralysis after stroke}

Wang Xiangming [6] selection of electric Vitalstim low frequency stimulation (America), The instrument output bidirectional square wave current, $700 \mathrm{~ms}$ wave width, fixed frequency range $30-80 \mathrm{~Hz}, 0-25 \mathrm{~m}$ peak output current A, dual channel, surface electrodes were placed on the buccal branch of facial nerve, ramus, traveling along the nerve was laid. Stimulation time $1 \mathrm{H}$ every day, for the treatment of $10 \mathrm{~d}$, the intensity of stimulation patients feel scratching feeling appropriate, $6-17.5 \mathrm{~m}$ buccal branch of $\mathrm{A}$, the average was $11.27 \pm 3.07 \mathrm{M} \mathrm{A} ; 6.5-18 \mathrm{~m} \mathrm{~A}$ ramus, the average was $10.48 \pm 2.98 \mathrm{M}$ A. two $6-18 \mathrm{~m} \mathrm{~A}$, the average was $11.67 \pm 3.18 \mathrm{~m} \mathrm{~A}$., and the facial nerve function training, the results of MHBN facial nerve function score were significantly improved $(\mathrm{P}<0.05)$.

\section{Treatment of urinary retention after stroke}

Yang Xiaohui [7] application of KT-90A nerve injury treatment, two to $80 * 40 \mathrm{~mm}$ conductive silicone rubber plate electrodes were placed in the hole in the pole and diuretic, pangguangshu, electrode plate is sheathed by hot water soaked cotton jacket, covered with waterproof plastic, and fixed with medical tape to output two-way asymmetric polarity square wave, pulse frequency $1.6 \mathrm{~Hz}$, using partial denervation prescription, the current output intensity increases gradually increased until the patient tolerance, in principle does not cause pain is appropriate, each treatment at $30 \mathrm{~min}$. for 30 patients, 22 cases were cured, 5 cases effective, 3 cases ineffective, there are the efficiency of $90 \%$.

\section{Functional electrical stimulation}

FES is low frequency pulsed current with certain intensity, to stimulate one or more groups of muscles by preset programs, thereby inducing muscle movement or voluntary muscle movement simulation of normal, in order to improve or restore stimulate the muscles or muscle function to the [8]. therapy is the most important feature is the can to produce immediate limb functional activities of [9].FES clinical application is the most widely used as a support for functional training for paralyzed people.

\section{Treatment of limb paralysis after stroke}

$\mathrm{Li} \mathrm{Li} \mathrm{[10]} \mathrm{FES} \mathrm{and} \mathrm{give} \mathrm{the} \mathrm{hemiplegic} \mathrm{limb} \mathrm{function} \mathrm{training,} \mathrm{the} \mathrm{stimulation} \mathrm{frequency} \mathrm{is} 033 \sim 150 \mathrm{~Hz}$, the 20 30 min of each treatment, after treatment, the lower limb motor function, balance function and ADL ability were significantly improved. Liu Zhongliang found that the function of electricity stimulation combined with early rehabilitation of patients, improve the walking and self-care ability, improve the quality of life, prevention of secondary damage has important significance of [11]. Fang 
Hua also found that early treatment of FES treatment group showed lower extremity Fugl-Meyer integral and ADL Barthel index and not after the patients had statistical significance, suggesting that early treatment with FES stroke patients, can help patients more quickly restore limb function [12].

Treatment of dysphagia after stroke

Wu Shaoling [13] using the United States VitalStim electrical stimulation, the treatment of dysphagia after stroke patients, stimulus intensity was $5-11 \mathrm{~m} \mathrm{~A}$, the frequency of the pulse output is $30-80 \mathrm{~Hz}$, the output waveform for double phase square, maximum intensity of $25 \mathrm{~m} \mathrm{~A}$ (SD + 10\%), wave width is $700 \mathrm{~ms}, 2$ output channels, each output channel has 2 electrodes, 4 electrodes. The intensity of treatment and treatment of electrodes placed by the therapist according to the patient's feeling, dysphagia type and severity adjusted. The results showed that electrical stimulation group SSA score and $\mathrm{Sa} \mathrm{O}_{2}$ values were lower than before treatment decreased, nerve muscle stimulation can improve the swallowing function of patients with dysphagia after stroke.

\section{Summary}

In summary, TENS treatment of stroke patients to alleviate spasticity, improve limb function; FES can improve the paralyzed limb blood circulation, delay and prevent muscle atrophy, adjust flexor extensor muscle tension, balance between groups, maintain and improve the joint range of motion and promote functional recovery of [4], also inspired by NMES repeated limb movement and learning activities with the efficacy of [14-16].

Neuromuscular electrical stimulation is an effective, economic and practical effect of rehabilitation treatment. The effect of frequency, location and intensity factors such as stimulation, to set the stimulus program according to the purpose of the treatment and the treatment site before the treatment, the operation needs to reflect the individual principle, not with a prescription to solve all their problemsof all patients therefore[17], such therapy at the stimulation site and stimulation parameters (time, intensity, frequency) large multicenter studies also need.

\section{Acknowledgements}

This work was financially supported by the Jilin Provincial Department of Education Commission (2015153).

\section{References}

[1] Sheffler L,Chae J.Neuromuscular electrical stimulation in aneurorehabilitation.J.Muscle Nerve,35:562-590(2007)

[2] Guo Youhua, Yan Tiebin ,Christina WY Hui-chan. Research progress on the mechanism of low frequency electrical stimulation in the treatment of stroke hemiplegia.J.Chinese Journal of Rehabilitation Medicine, 20 (2): 156-158(2005)

[3] Shen Guangyu, Qian cuoquan, Cai Kefu.. Neuromuscular electrical stimulation on stroke early motor function recovery.J. Journal of Nantong University (Medical Science Edition),28 (5): 410-411(2008)

[4] Zhao Yulan. The effect of electrical stimulation on motor function in patients with cerebral infarction. J. Chinese Journal of physical medicine and rehabilitation, 26: 575-576(2004)

[5] Wang Dongyan. Study on the improvement of wrist function after stroke by low frequency pulse electric acupoint therapy.J. Chinese Journal of rehabilitation medicine, 25 (3) (2008)

[6] Hou Ying, Li Wen. Therapeutic effects of low frequency electrical stimulation on the treatment of post stroke paralysis.J. Chinese Journal of rehabilitation medicine, 24 (3) (2009) 
[7] Yang Xiaohui, Zhang Shuli,Luo Hongmei. Low-frequency pulse therapy on clinical observation of urinary retention after stroke.J. China Journal of Gerontology, 28 (11)(2008)

[8] Rushton DN. Functional electrical stimulation. Physiol Meas, 18:241 -275( 1997)

[9] Levin MF, Hui-Chan CWY.Conventional and acupuncture-like transcu-taneouselectrical nerve stimulation excite similar afferent fibers. Arch Phys Med Rehabi,74: 54-60(1993)

[10] Li Li, Yuan Jiaqi, Zhang Chenyi. Clinical observation of functional electrical stimulation and functional training of lower limbs in hemiplegic patients .J. Chinese Journal of physical medicine and rehabilitation,22: 18-19(2000)

[11] Liu Zhongliang, Song Lin, et al. Effects of functional electrical stimulation on upper limb motor function recovery in stroke patients with hemiplegia.J. rehabilitation of China,20 (1): 52-53(2005)

[12] Fang Hua, Zhou Zhixian, Liu Guifen. The effect of functional electrical stimulation on motor function recovery in stroke patients.J.Chinese Journal of clinical rehabilitation,8 (16):3004-3005. , (2004)

[13] Wu Shaoling, Yan Wei, Ma Chao, et al. The effect of neuromuscular electrical stimulation combined with functional training on dysphagia after stroke.J.Chinese Journal of physical medicine and rehabilitation,29537-539(2007)

[14] Chae J.Neuromuscular electrical stimulation for motor relearning in hemiparesis.J.Phys Med Rehabil Clin N Am,14(Suppl):S93-109(2003)

[15] Gad A.Use of neuromuscular electrical stimulation in neureorehabilitation:A challenge to all.J .Rehabil Res Develop,40(6):9-12(2003)

[16] Sheffler L,Chae J.Neuromuscular electrical stimulation in aneurorehabilitation.J.Muscle Nerve,35:562-590(2007)

[17] Yan Tiebin actively promotes the application of neuromuscular electrical stimulation in the treatment of central nervous system injury. Chinese Journal of rehabilitation medicine,22 (10): $865(2007)$ 